The Free Internet Journal

for Organic Chemistry
Paper

Arkivoc 2019, part iii, 40-52

\title{
Routes to N-glycinamide oxazolidinone derivatives: The reaction of 4- trityloxymethyl-3-oxa-1-azabicyclo[3.1.0]hexan-2-one with active halides
}

\author{
Zilong Zheng and Stephen C. Bergmeier* \\ Department of Chemistry \& Biochemistry, Clippinger Laboratories, Ohio University, Athens, OH, 45701, USA \\ Email: bergmeis@ohio.edu
}

To Professor George Kraus for bringing to life my interest in organic chemistry

Received 11-12-2018

Accepted 01-19-2019

Published on line 01-26-2019

\section{Abstract}

$\mathrm{N}$-Glycinamide oxazolidinones are key intermediates for the synthesis of a new class of peptidomimetics. We report, herein, the synthesis of a series of 3,4,5-trisubstituted oxazolidinones with a glycine residue at N-3 of the oxazolidinone ring. Substituents at C-4 and C-5 contain substitution capabilities which are amenable for the introduction of additional peptide chains. The synthesis of the key N-Glycinamide oxazolidinone is made possible through two reactions; a tandem aziridine ring opening/oxazolidinone alkylation and, subsequently, a fused-lactone ring opening by an amine which leads to the N-Glycinamide oxazolidinones.<smiles>[R]NC(=O)CN1C(=O)O[C@H](CO[Te][C]CC)[C@@H]1CO</smiles>

Keywords: Fused-ring aziridines, oxazolidinones, peptidomimetics, lactones, bicyclic 


\section{Introduction}

Peptides continue to be a source of inspiration for the design of therapeutic molecules. Modification of the peptide backbone to generate peptidomimetics provides a route to use both the sequence information of peptides as well as stability and structural constraints imposed by the synthetic components. ${ }^{1-3}$ Oxazolidinones and related linkers have been used previously to prepare a variety of peptidomimetics. The use of both the oxazolidin-4-one type rings $(\mathbf{1})^{4}$ as well as oxazolidine-2-one rings $(\mathbf{2})^{5-9}$ (Figure 1 ) have been reported. In both cases, the oxazolidinone ring has generally been used to induce formation of secondary structures. In the reported oxazolidine-2-one peptidomimetics, the oxazolidinone ring is acylated with an amino acid or peptide chain, while the 4-position contains a carboxylate group used to acylate the nitrogen of a second peptide chain.
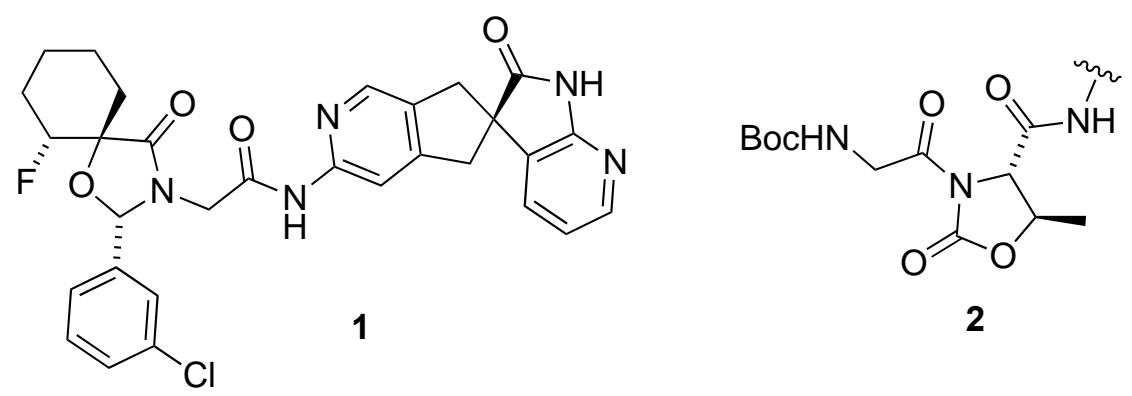

Figure 1. Examples of oxazolidinone containing peptidomimetics.

We were interested in generating a unique set of oxazolidinone-based peptidomimetics such as $\mathbf{3}$ in which the nitrogen on the oxazolidinone ring would be alkylated with a peptide chain. A substituted methyl group ( $X$ $=\mathrm{O}, \mathrm{N}$ or halogen) on the 4-position would allow for attachment of an additional peptide chain. The hydroxymethyl group at C-5 would be available to attach additional functionality to aid in solubility or target identification. It was anticipated that $\mathbf{3}$ could be prepared from an oxazolidinone such as 4 through modification of the C-4 hydroxymethyl group. Oxazolidinone 4 could be available via an aziridine ring opening/alkylation sequence starting with fused ring aziridine 5 (Scheme 1).
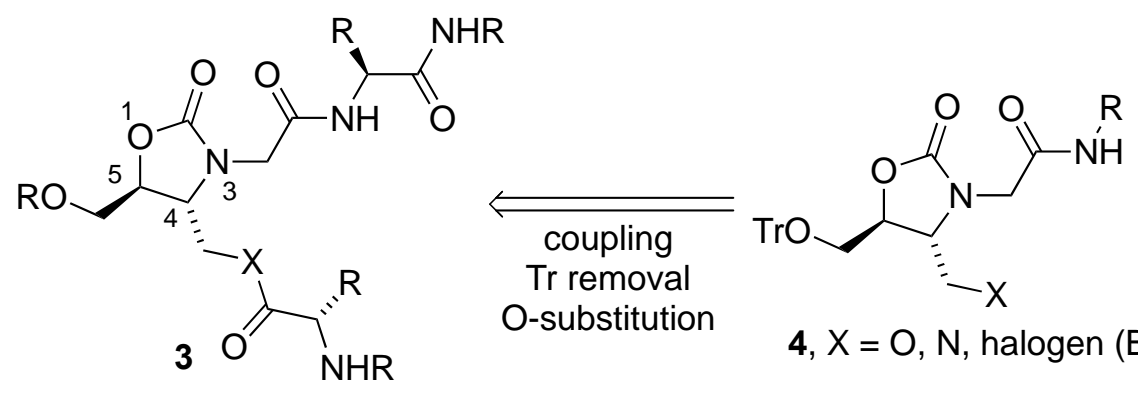

O-substitution

4, $\mathrm{X}=\mathrm{O}, \mathrm{N}$, halogen $(\mathrm{Br}, \mathrm{I})$

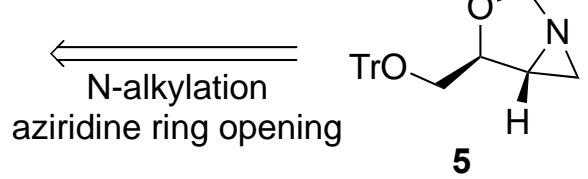

Scheme 1. Proposed route for preparing oxazolidinone-based peptidomimetics.

We have previously shown the utility of aziridines such as $5^{\mathbf{1 0}}$ for the preparation of natural products such as bestatin ${ }^{11}$, as well as inhibitors of the transcription read-through in an RNA riboswitch. ${ }^{10,12-14}$ We were intrigued with the possibility of an initial reaction of the aziridine of $\mathbf{5}$ with a reactive alkyl halide that might be followed by aziridine ring opening with a nucleophile to provide $\mathbf{4}$ or related oxazolidinones. Similar types of 
reactions have been reported by Pinhas et al. ${ }^{15}$ The addition reaction of an $\mathrm{N}$-acylaziridine (e.g., 5) and reactive halides has, however, remained relatively unexplored. Consequently, we propose that reaction of aziridine $\mathbf{5}$ with a reactive halide should result in the $\mathrm{N}$-alkylation of the oxazolidinone and concomitant ring opening.

\section{Results and Discussion}

Based on this hypothesis, the addition reaction of the fused-ring aziridine (5) and an iodoacetate was examined. Reaction of aziridine 5 with t-butyl iodoacetate (6a) in refluxing $\mathrm{CH}_{3} \mathrm{CN}$ provided a moderate yield of the desired oxazolidinone $7 a$ (Scheme 2).
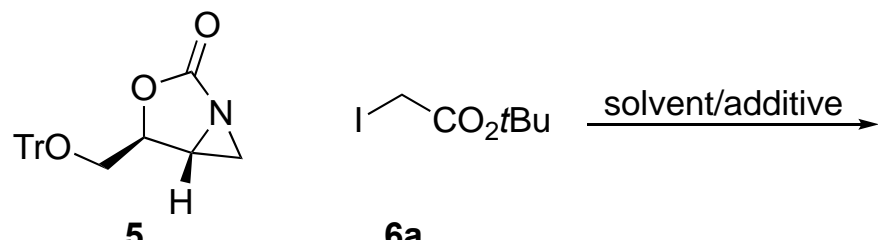

$6 a$

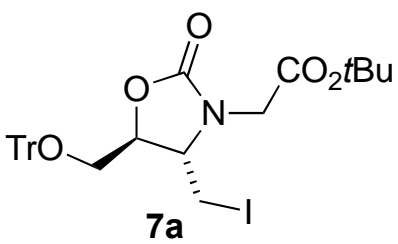

Scheme 2. Reaction of aziridine $\mathbf{5}$ with an iodoacetate.

Several solvents, additives and temperatures were examined in order to optimize the reaction conditions (Table 1). Using dried $\mathrm{CH}_{3} \mathrm{CN}$, as opposed to the slightly wet $\mathrm{CH}_{3} \mathrm{CN}$ from the reagent bottle, provided a moderate increase in yield of the reaction. Use of a more polar solvent such as DMF provided a poorer yield of the product (entry 3), while THF provided $7 \mathrm{a}$ in a similar yield as wet $\mathrm{CH}_{3} \mathrm{CN}$. Neither acetone nor dioxane provided any of the expected oxazolidinone $7 \mathbf{a}$.

Table 1. Reaction-optimization conditions for the reaction of fused-ring aziridine and iodoacetate

\begin{tabular}{llll}
\hline Entry & Solvent/Additive & Conditions & Yield (\%) \\
\hline 1 & $\mathrm{CH}_{3} \mathrm{CN}$ & Reflux & 51 \\
2 & $\mathrm{CH}_{3} \mathrm{CN}^{\mathrm{a}}$ & Reflux & 61 \\
3 & $\mathrm{DMF}$ & $10{ }^{\circ} \mathrm{C}$ & 28 \\
4 & $\mathrm{THF}$ & Reflux & 42 \\
5 & Acetone & Reflux & 0 \\
6 & $1,4-$ Dioxane & Reflux & 0 \\
7 & $\mathrm{CH}_{3} \mathrm{CN}^{\text {a }} / \mathrm{Nal}$ & Reflux & 83 \\
\hline
\end{tabular}

${ }^{\mathrm{a}}$ Dried by distillation.

Mechanistically, the reaction could proceed via an initial iodide-mediated aziridine-ring opening leading to intermediate $\mathbf{A}$, which would be followed by an alkylation to provide 7a, as shown in Scheme 3. Alternatively, the alkylation of the aziridine with 6 a could occur first to provide the intermediate aziridinium $\mathbf{B} .{ }^{16-18}$ This would be followed by ring opening with iodide to provide 7a. Additional Nal resulted in a significant increase in overall yield of the alkylated oxazolidinone, suggesting that the reaction proceeds via an initial ring opening followed by alkylation. 


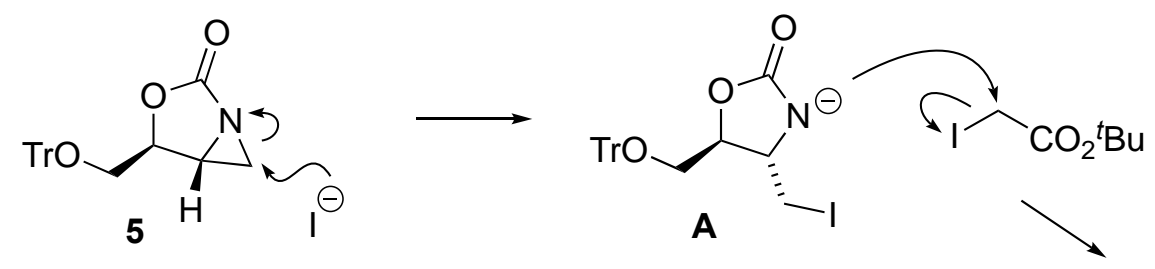

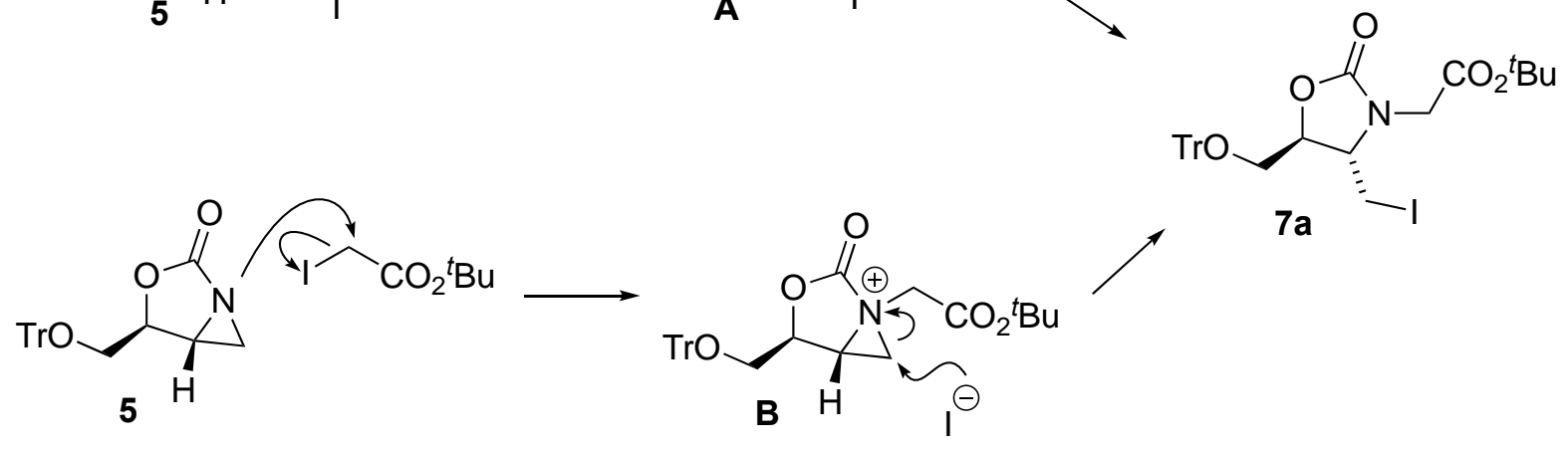

Scheme 3. Possible mechanistic rationale for the reaction of $\mathbf{5}$ with an iodoacetate.

With good reaction conditions in hand, the scope of the reaction was studied with the hope that amide derivatives such as $\mathbf{3}$ could be directly prepared. Additional esters, such as methyl and ethyl iodoacetates, readily reacted with the fused-ring aziridine to produce the desired oxazolidinone products $7 \mathbf{b}$ and $7 \mathbf{c}$ in excellent yields (Scheme 4; Table 2).
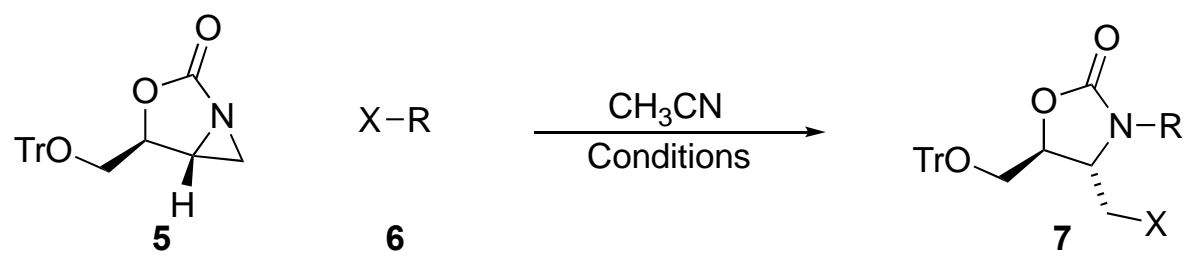

Scheme 4. General reaction of aziridine (5) with activated halides.

The reactions of the fused-ring aziridine and a series of amides were completely unsuccessful. Neither $\mathrm{N}, \mathrm{N}$-dimethyl iodoamide, $\mathrm{N}$-methyl iodoamide or $\mathrm{N}$-benzyl iodoamide provided any of the desired product. Interestingly, the reaction of $\mathrm{N}, \mathrm{N}$-dimethyl iodoamide (entry 4) did provide a moderate yield of 4-iodomethyl5-trityloxymethyl-oxazolidin-2-one (7d), again, suggesting that ring opening of the aziridine by the iodide ion was a key step of the reaction. The $\alpha$-iodo ketone, iodo-1-phenylethanone, provided product $7 \mathrm{~g}$ in $54 \%$ yield (entry 7). lodoacetonitrile did not generate any addition product (entry 8). Activated halides, such as benzyl iodide $(87 \%$, entry 9$)$, also provided the addition product.

With the success of the reactions of the fused-ring aziridines with the iodides, the reactions with select bromides were also examined. Tert-butylbromoacetate readily underwent reaction with aziridine $\mathbf{5}$ to provide the oxazolidinone $\mathbf{7 j}$ in $\mathbf{7 4 \%}$ yield, which was somewhat lower than the reaction with the corresponding iodide. Unlike the reactions with iodides, the addition of $\mathrm{NaBr}$ to the reaction did not improve the yield, and, instead, led to decomposition of the starting material. As with the iodoamides, the bromoamide (entry 12) did not provide any of the expected product. The reaction of benzyl bromide with aziridine $\mathbf{5}$ gave a lower yield of the oxazolidinone product than the corresponding iodide (entry 9 vs. entry 13). The use of $\alpha$-tosyloxy-methyl acetate was also unsuccessful in providing the alkylated oxazolidinone product (entry 14). 
Table 2. Reactions of aziridine (5) with activated halides

\begin{tabular}{|c|c|c|c|c|c|c|}
\hline Entry & Compound \# & $x$ & $\mathrm{R}$ & Additive & Conditions & Yield (\%) \\
\hline 1 & $7 a$ & 1 & $\mathrm{CH}_{2} \mathrm{CO}_{2}{ }^{t} \mathrm{Bu}$ & $\mathrm{Nal}$ & Reflux & 83 \\
\hline 2 & $7 b$ & I & $\mathrm{CH}_{2} \mathrm{CO}_{2} \mathrm{Me}$ & $\mathrm{Nal}$ & Reflux & 90 \\
\hline 3 & 7c & I & $\mathrm{CH}_{2} \mathrm{CO}_{2} \mathrm{Et}$ & Nal & Reflux & 99 \\
\hline 4 & $7 d$ & I & $\mathrm{CH}_{2} \mathrm{CON}\left(\mathrm{CH}_{2}\right)_{4}$ & $\mathrm{Nal}$ & Reflux & $0^{a}$ \\
\hline 5 & $7 e$ & I & $\mathrm{CH}_{2} \mathrm{CONHMe}$ & Nal & Reflux & 0 \\
\hline 6 & $7 f$ & I & $\mathrm{CH}_{2} \mathrm{CONHBn}$ & Nal & Reflux & 0 \\
\hline 7 & $7 g$ & I & $\mathrm{CH}_{2} \mathrm{COPh}$ & Nal & Reflux & 54 \\
\hline 8 & 7h & I & $\mathrm{CH}_{2} \mathrm{CN}$ & Nal & Reflux & 0 \\
\hline 9 & $7 \mathbf{i}$ & I & $\mathrm{CH}_{2} \mathrm{Ph}$ & Nal & Reflux & 87 \\
\hline 10 & $7 \mathbf{j}$ & $\mathrm{Br}$ & $\mathrm{CH}_{2} \mathrm{CO}_{2}{ }^{t} \mathrm{Bu}$ & & Reflux & 74 \\
\hline 11 & $7 \mathbf{j}$ & $\mathrm{Br}$ & $\mathrm{CH}_{2} \mathrm{CO}_{2}{ }^{t} \mathrm{Bu}$ & $\mathrm{NaBr}$ & Reflux & 0 \\
\hline 12 & $7 k$ & $\mathrm{Br}$ & $\mathrm{CH}_{2} \mathrm{CON}\left(\mathrm{CH}_{2}\right)_{4}$ & & Reflux & 0 \\
\hline 13 & 기 & $\mathrm{Br}$ & $\mathrm{CH}_{2} \mathrm{Ph}$ & & Reflux & 53 \\
\hline 14 & $7 m$ & OTs & $\mathrm{CH}_{2} \mathrm{CO}_{2} \mathrm{Me}$ & & Reflux & 0 \\
\hline
\end{tabular}

a 4-lodomethyl-5-trityloxymethyl-oxazolidin-2-one was obtained in $74 \%$ yield.

Given the lack of success using $\alpha$-halo amides in the iodide-mediated ring-opening/alkylation sequence, an alternate route to the desired amides (4) was designed. The reaction of an amine with a lactone such as 8 could provide a facile route to a variety of glycinamide substituted oxazolidinones (4). A silver-catalyzed cyclization of the iodomethyl group with the t-butyl ester might be expected to provide the bicyclic lactone (8). ${ }^{19}$ It was hoped that the t-butyl ester would react with the incipient cation formed by reaction of the iodide and silver salt. This would then release the t-butyl cation and provide $\mathbf{8}$. Subsequent opening of $\mathbf{8}$ with an amine would provide the glycine substituted oxazolidinone 4 as shown in Scheme 5.
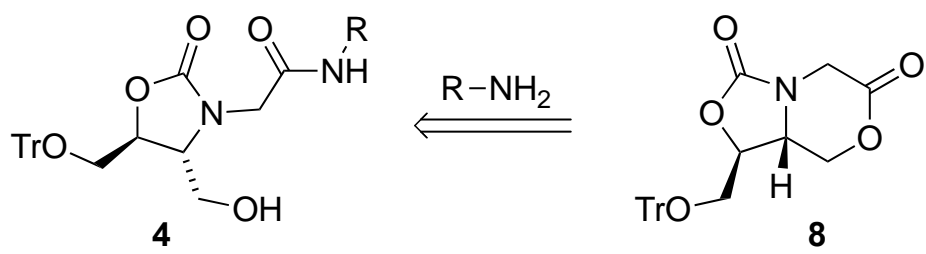

Ag salt

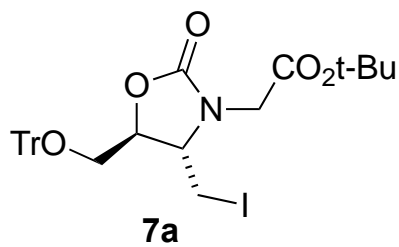

Scheme 5. Proposed route to glycinamide 4 from iodide 7a.

Multiple attempts to generate the lactone $\mathbf{8}$ directly from $\mathbf{7 a}$ were unsuccessful. Multiple silver salts, solvent, and temperatures were investigated without success. In order to carry out a formation of the lactone 8, an alternate approach from the corresponding carboxylic acid was examined (Scheme 6).
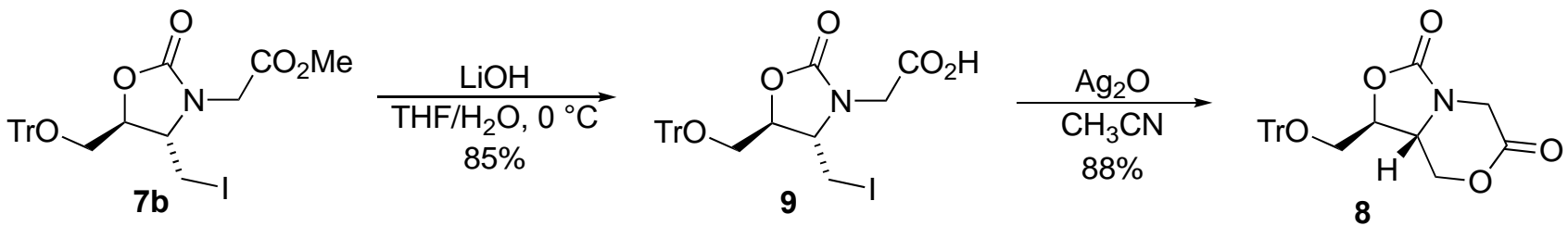

Scheme 6. Conversion of iodide 7 to bicycle 8. 
Treatment of ester $\mathbf{7 b}$ with $\mathrm{LiOH}$ in $\mathrm{THF} / \mathrm{H}_{2} \mathrm{O}$ at $0{ }^{\circ} \mathrm{C}$ provided acid 9 in excellent yield. With the carboxylic acid 9 in hand, the cyclization reaction promoted by different silver salts was studied. ${ }^{19}$ Silver trifluoroacetate ${ }^{20}$ led only to the loss of the trityl group. Adding $\mathrm{Na}_{2} \mathrm{CO}_{3}$ to the silver trifluoroacetate to buffer the reaction did not lead to product either. Silver acetate provided lactone 8 in only $17 \%$ yield, again with significant loss of the trityl group. Addition of $\mathrm{Na}_{2} \mathrm{CO}_{3}$ did not help since none of the expected lactone was obtained under these conditions. Ultimately, silver oxide was found to be the best silver source, with a $78 \%$ yield of the product in $\mathrm{CH}_{2} \mathrm{Cl}_{2}$. Upon changing the solvent to $\mathrm{CH}_{3} \mathrm{CN}$, the yield was increased to $88 \%$. ${ }^{10}$

With the key bicyclic lactone $\mathbf{8}$ in hand, the reactions of the lactone moiety with amines could be examined. Gratifyingly, it was found that the fused-ring lactone moiety 8 could readily react with primary amines to provide the desired products 4 in excellent yields (89\%-100\%) (Scheme 7; Table 3).
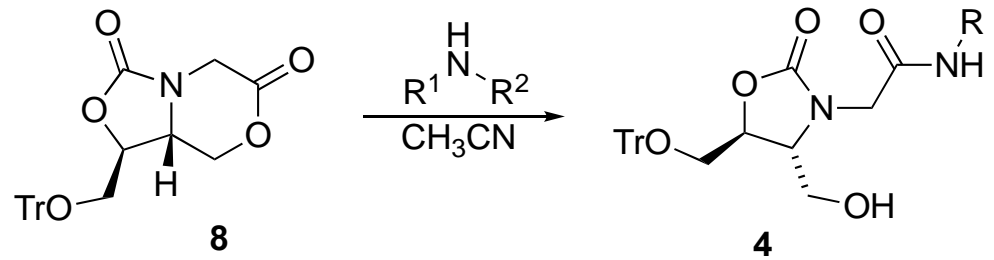

Scheme 7. Reaction of lactone 8 with a series of amines.

Simple primary amines such as methyl, isopropyl, isobutyl, allyl, and benzyl, and 4-methoxybenzylamine all provided amides $\mathbf{4 a - 4 f}$ in excellent yield. Methoxylethyl amine, as well as hydroxyethyl amine, provided amides $\mathbf{4 g}$ and $\mathbf{4 h}$, respectively. Glycine derivatives, such as glycine $\mathrm{N}$-methyl amide and glycine methyl ester, both provided the corresponding amide derivatives $4 \mathbf{i}$ and $\mathbf{4} \mathbf{j}$ in excellent yields. The use of the secondary amine, dibutyl amine, provided none of the expected amide.

Table 3. Results of reactions of lactone 8 with amines

\begin{tabular}{llll}
\hline Entry & Product & $\mathbf{R}^{\mathbf{1}}, \mathbf{R}^{\mathbf{2}}$ & Yield (\%) \\
\hline 1 & $\mathbf{4 a}$ & $\mathrm{R}^{1}=\mathrm{CH}_{3}, \mathrm{R}^{2}=\mathrm{H}$ & 100 \\
2 & $\mathbf{4 b}$ & $\mathrm{R}^{1}=\mathrm{iPr}, \mathrm{R}^{2}=\mathrm{H}$ & 100 \\
3 & $\mathbf{4 c}$ & $\mathrm{R}^{1}=\mathrm{CH}_{2} \mathrm{CH}\left(\mathrm{CH}_{3}\right)_{2}, \mathrm{R}^{2}=\mathrm{H}$ & 90 \\
4 & $\mathbf{4 d}$ & $\mathrm{R}^{1}=\mathrm{allyl}, \mathrm{R}^{2}=\mathrm{H}$. & 100 \\
5 & $\mathbf{4 e}$ & $\mathrm{R}^{1}=\mathrm{Bn}^{2} \mathrm{R}^{2}=\mathrm{H}$ & 93 \\
6 & $\mathbf{4 f}$ & $\mathrm{R}^{1}=\mathrm{CH}_{2}\left(4-\mathrm{OCH}_{3} \mathrm{C}_{6} \mathrm{H}_{4}\right) \mathrm{R}^{2}=\mathrm{H}$ & 90 \\
7 & $\mathbf{4 g}$ & $\mathrm{R}^{1}=\mathrm{CH}_{2} \mathrm{CH}_{2} \mathrm{OCH}_{3}, \mathrm{R}^{2}=\mathrm{H}$ & 100 \\
8 & $\mathbf{4 h}$ & $\mathrm{R}^{1}=\mathrm{CH}_{2} \mathrm{CH}_{2} \mathrm{OH}, \mathrm{R}^{2}=\mathrm{H}$ & 89 \\
9 & $\mathbf{4 i}$ & $\mathrm{R}^{1}=\mathrm{CH}_{2} \mathrm{C}(\mathrm{O}) \mathrm{NHCH}_{3}, \mathrm{R}^{2}=\mathrm{H}$ & 90 \\
10 & $\mathbf{4 j}$ & $\mathrm{R}^{1}=\mathrm{CH}_{2} \mathrm{C}(\mathrm{O}) \mathrm{OCH}_{3}, \mathrm{R}^{2}=\mathrm{H}$ & 96 \\
11 & $\mathbf{4 k}$ & $\mathrm{R}^{1}=\mathrm{R}^{2}=\mathrm{nBu}$ & 0 \\
\hline
\end{tabular}

The free alcohol resulting from the amine opening of the lactone was subsequently acylated to demonstrate a route to potential peptidomimetics. It was found that Steglich-esterification conditions worked quite well to provide a small series of ester derivatives from amide 4a (Scheme 8). 2-Methyl propanoic acid and 4-Pentynoic acid provided the expected products $10 \mathrm{a}$ and $10 \mathrm{~b}$ in yields of 81 and $85 \%$, respectively. The amino acid Boc-glycine provided $10 \mathrm{c}$ in $77 \%$ yield. 

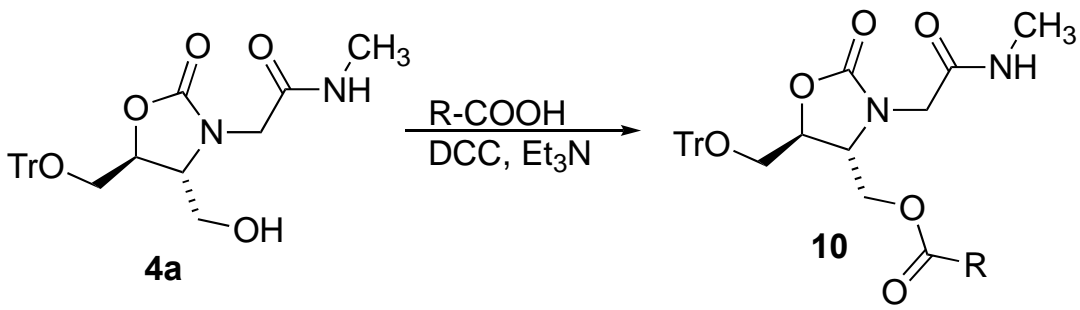

10a, $\mathrm{R}=\mathrm{iPr}, 81 \%$

10b, $\mathrm{R}=\left(\mathrm{CH}_{2}\right)_{2} \mathrm{CCH}, 85 \%$

10c, $\mathrm{R}=\mathrm{CH}_{2} \mathrm{NHBoc}, 77 \%$

Scheme 8. Acylation of alcohol 4a.

\section{Conclusions}

The synthesis of a novel class of oxazolidinone peptidomimetic has been described. This method uses a new reaction of fused-ring aziridines with activated halides to generate $\mathrm{N}$-alkyl and $\mathrm{N}$-acyl oxazolidinones from a fused-ring aziridine. This ring-opened product has been converted to a bicyclic lactone which readily reacts with primary amines to generate oxazolidinone glycinamides. Further studies on the synthesis of peptidomimetics based on these results will be reported in due course.

\section{Experimental Section}

General. ${ }^{1} \mathrm{H}$ NMR (300 MHz or $500 \mathrm{MHz}$ ) and ${ }^{13} \mathrm{C}$ NMR spectra were measured on a Bruker Avance $300 \mathrm{MHz}$ or $500 \mathrm{MHz} N M R$ spectrometer with TMS used as the internal standard. High Resolution Mass Spectrometry (HRMS) measurements were performed on a Bruker 12 Tesla APEX -Qe FTICR-MS or Q Exactive Plus from Thermo Scientific. $\mathrm{CH}_{2} \mathrm{Cl}_{2}$ and THF were dried using a SOLVTEK solvent purification system. $\mathrm{CH}_{3} \mathrm{CN}$ was distilled from $\mathrm{CaH}_{2}$ and stored over molecular sieves. All amines were purified by distillation prior to use. Aziridine $\mathbf{5}$ was prepared as previously reported. ${ }^{10}$ All reactions were conducted under an atmosphere of argon. All other reagents were used as purchased. Chromatography refers to flash chromatography on silica gel according to the method of Still. ${ }^{21}$ The $R^{*} / S^{*}$ notation is intended to indicate relative stereochemistry. ${ }^{22}$

General Procedure A. Reaction of bicyclic lactone (8) with amines: The desired amine (200 mol\%) was added to a solution of lactone compound 8 in $\mathrm{CH}_{3} \mathrm{CN}(0.05 \mathrm{M})$. The mixture was stirred at room temperature until the reaction was complete as determined by TLC. The solvent was removed and compound 4 was purified via column chromatography $\left(\mathrm{CH}_{2} \mathrm{Cl}_{2}: \mathrm{MeOH} ; 15: 1\right)$.

2-((4R*,5 $\left.\left.S^{*}\right)-4-H y d r o x y m e t h y l-2-o x o-5-t r i t y l o x y m e t h y l-o x a z o l i d i n-3-y l\right)-N$-methylacetamide (4a). Compound 4a (43 mg, 96\%) was obtained as a white solid from 8 (42 mg, $0.1 \mathrm{mmol}$ ) and methylamine (6.2 mg methylamine in $12.4 \mathrm{mg}$ of methanol, $0.2 \mathrm{mmol}$ ) following General Procedure $\mathrm{A}$; $\mathrm{mp} 179-181{ }^{\circ} \mathrm{C}$; ${ }^{1} \mathrm{H} \mathrm{NMR}$ $\left(\mathrm{CDCl}_{3}, 500 \mathrm{MHz}\right) \delta$ 7.48-7.46 (m, 6H), 7.35-7.32 (m, 6H), 7.28-7.25 (m, 3H), 6.14 (br s, 1H), 4.83-4.81 (m, 1H), 4.59 (q, J $4.5 \mathrm{~Hz}, 1 \mathrm{H}), 4.20$ (d, J16.0 Hz, 1H), 3.72 (dd, J 3.0, $13.0 \mathrm{~Hz}, 1 \mathrm{H}), 3.60-3.57(\mathrm{~m}, 2 \mathrm{H}), 3.46-3.43(\mathrm{~m}, 2 \mathrm{H})$, 3.19 (dd, J 4.0, $10.5 \mathrm{~Hz}, 1 \mathrm{H}), 2.81$ (d, J 4.5, 3H). ${ }^{13} \mathrm{C} \mathrm{NMR}\left(\mathrm{CDCl}_{3}, 125 \mathrm{MHz}\right) \delta 170.0,158.6,143.3,128.5,128.1$, 127.4, 87.0, 75.3, 63.9, 61.9, 60.6, 46.3, 26.5. HRMS-ESI: $m / z[M+N a]^{+}$calcd for $\mathrm{C}_{27} \mathrm{H}_{28} \mathrm{~N}_{2} \mathrm{O}_{5} \mathrm{Na}^{+}: 483.1890$; Found: 483.1891.

2-((4R*,5S*)-4-Hydroxymethyl-2-oxo-5-trityloxymethyl-oxazolidin-3-yl)-N-isopropylacetamide (4b). Compound 4b (40 mg, yield 84\%) was obtained as a white solid from 8 (42 mg, $0.1 \mathrm{mmol}$ ) and isopropyl amine (12 mg, 0.2 
mmol) following General Procedure A; mp $128-130{ }^{\circ} \mathrm{C} ;{ }^{1} \mathrm{H}$ NMR $\left(\mathrm{CDCl}_{3}, 500 \mathrm{MHz}\right) \delta$ 7.48-7.46 (m, 6H), 7.35-7.32 (m, $6 \mathrm{H}), 7.28-7.25(\mathrm{~m}, 3 \mathrm{H}), 4.61(\mathrm{q}, J 4.5 \mathrm{~Hz}, 1 \mathrm{H}), 4.17(\mathrm{~d}, J 17.0 \mathrm{~Hz}, 1 \mathrm{H}), 4.01(\mathrm{sept}, J 6.5 \mathrm{~Hz}, 1 \mathrm{H}), 3.81(\mathrm{~d}, J 17.0 \mathrm{~Hz}, 1 \mathrm{H})$, 3.73-3.67 (m, 2H), 3.42-3.36 (m, 2H), $3.22(\mathrm{dd}, J 4.5,10.5 \mathrm{~Hz}, 1 \mathrm{H}), 1.14(\mathrm{~d}, J 6.5 \mathrm{~Hz}, 3 \mathrm{H}), 1.13(\mathrm{~d}, J 6.5 \mathrm{~Hz}, 3 \mathrm{H}) .{ }^{13} \mathrm{C}$ NMR (Acetone- $\left.d_{6}, 125 \mathrm{MHz}\right) \delta 170.7,159.5,145.6,130.4,129.7,128.9,88.3,76.7,66.3,62.9,61.7,47.4,43.2,23.5$. HRMS-ESI: $m / z$ [M+Na]+ calcd for $\mathrm{C}_{29} \mathrm{H}_{32} \mathrm{~N}_{2} \mathrm{O}_{5} \mathrm{Na}+$ : 511.2203 ; Found: 511.2213 .

2-((4R*,5S*)-4-Hydroxymethyl-2-oxo-5-trityloxymethyl-oxazolidin-3-yl)-N-isobutylacetamide (4c). Compound 4c (300 mg, 90\%) was obtained as white solid from compound 8 (290 mg, $0.7 \mathrm{mmol}$ ) and isobutyl amine (102 mg, 1.4 mmol) following General Procedure A; mp 128-131 ${ }^{\circ} \mathrm{C} ;{ }^{1} \mathrm{H}$ NMR (Acetone-d $\left.\mathrm{d}_{6}, 500 \mathrm{MHz}\right) \delta 7.16$ (br s, 1H), 7.48-7.46 $(\mathrm{m}, 6 \mathrm{H}), 7.38-7.33(\mathrm{~m}, 6 \mathrm{H}), 7.29-7.26(\mathrm{~m}, 3 \mathrm{H}), 5.16(\mathrm{dd}, J 4.0,10.0 \mathrm{~Hz}, 1 \mathrm{H}), 4.61(\mathrm{q}, J 4.5 \mathrm{~Hz}, 1 \mathrm{H}), 4.22(\mathrm{~d}, J 17.0 \mathrm{~Hz}$, $1 \mathrm{H}), 3.88(\mathrm{~d}, J 17.0 \mathrm{~Hz}, 1 \mathrm{H}), 3.74-3.70(\mathrm{~m}, 2 \mathrm{H}), 3.42-3.64(\mathrm{~m}, 2 \mathrm{H}), 3.23(\mathrm{dd}, J 4.5,10.5 \mathrm{~Hz}, 1 \mathrm{H}), 3.10-3.04(\mathrm{~m}, 2 \mathrm{H})$, 1.83-1.73 (m, 1H), 0.90 (d, J $6.5 \mathrm{~Hz}, 6 \mathrm{H}) .{ }^{13} \mathrm{C}$ NMR (Acetone-d $\left.\mathrm{d}_{6}, 125 \mathrm{MHz}\right) \delta 171.7,159.5,145.6,130.4,129.7,128.9$, 88.3, 76.7, 66.3, 62.9, 61.6, 48.4, 47.3, 21.3, 15.4. HRMS-ESI: $m / z[\mathrm{M}+\mathrm{Na}]^{+}$calcd for $\mathrm{C}_{30} \mathrm{H}_{34} \mathrm{~N}_{2} \mathrm{O}_{5} \mathrm{Na}^{+}: 525.2360$; Found: 525.2355

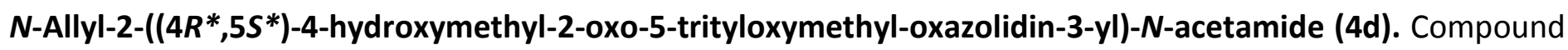
4d (46 mg, 98\%) was obtained as a white solid from compound 8 (42 mg, $0.1 \mathrm{mmol}$ ) and allyl amine (11 mg, $0.2 \mathrm{mmol}$ ) following General Procedure A; mp 152-154 ${ }^{\circ} \mathrm{C} ;{ }^{1} \mathrm{H} \mathrm{NMR}\left(\mathrm{CDCl}_{3}, 500 \mathrm{MHz}\right) \delta 7.40-7.39(\mathrm{~m}, 6 \mathrm{H}), 7.30$ $7.26(\mathrm{~m}, 6 \mathrm{H}), 7.23-7.20(\mathrm{~m}, 3 \mathrm{H}), 6.89(\mathrm{t}, J 5.0 \mathrm{~Hz}, 1 \mathrm{H}), 5.80-5.72(\mathrm{~m}, 1 \mathrm{H}), 5.17(\mathrm{dd}, J 1.0,17.0 \mathrm{~Hz}, 1 \mathrm{H}), 5.11(\mathrm{dd}, J$ 1.0, $10.0 \mathrm{~Hz}, 1 \mathrm{H}), 4.63(\mathrm{q}, J 4.5 \mathrm{~Hz}, 1 \mathrm{H}), 4.30(\mathrm{~d}, J 16.5 \mathrm{~Hz}, 1 \mathrm{H}), 3.89-3.83(\mathrm{~m}, 1 \mathrm{H}), 3.80-3.75(\mathrm{~m}, 1 \mathrm{H}), 3.70-3.69$ (m, $1 \mathrm{H}), 3.57(\mathrm{~d}, J 16.5 \mathrm{~Hz}, 1 \mathrm{H}), 3.53-3.52(\mathrm{~m}, 1 \mathrm{H}), 3.44-3.41(\mathrm{~m}, 1 \mathrm{H}), 3.40(\mathrm{dd}, J 4.5,10.5 \mathrm{~Hz}, 1 \mathrm{H}), 3.18(\mathrm{dd}, J$ 4.0, $10.5 \mathrm{~Hz}, 1 \mathrm{H}) .{ }^{13} \mathrm{C} \mathrm{NMR}\left(\mathrm{CDCl}_{3}, 125 \mathrm{MHz}\right) \delta 169.5,158.6,143.3,133.1,128.5,128.1,127.4,117.0,87.0$, 75.2, 63.9, 62.0, 60.6, 46.4, 42.3. HRMS-ESI: $\mathrm{m} / z[\mathrm{M}+\mathrm{Na}]^{+}$calcd for $\mathrm{C}_{29} \mathrm{H}_{30} \mathrm{~N}_{2} \mathrm{O}_{5} \mathrm{Na}^{+}:$509.2347; Found: 509.2052 . $\mathbf{N}$-Benzyl-2-((4R*,5S*)-4-hydroxymethyl-2-oxo-5-trityloxymethyl-oxazolidin-3-yl)acetamide (4e). Compound 4e (94 mg, 93\%) was obtained as a clear oil from compound 8 ( $81 \mathrm{mg}, 0.20 \mathrm{mmol}$ ) and benzyl amine (43 mg, $0.4 \mathrm{mmol})$ following General Procedure $\mathrm{A} ;{ }^{1} \mathrm{H} \mathrm{NMR}\left(\mathrm{CDCl}_{3}, 300 \mathrm{MHz}\right) \delta 7.40-7.37(\mathrm{~m}, 6 \mathrm{H}), 7.29-7.20(\mathrm{~m}, 15 \mathrm{H})$, 5.09-5.07 (m, 1H), $4.59(\mathrm{q}, J 4.8 \mathrm{~Hz}, 1 \mathrm{H}), 4.38(\mathrm{dd}, J 5.7,14.7 \mathrm{~Hz}, 1 \mathrm{H}), 4.29-4.21(\mathrm{~m}, 1 \mathrm{H}), 4.25(\mathrm{~d}, J 17.1 \mathrm{~Hz}, 1 \mathrm{H})$, 3.68-3.64 (m, 1H), $3.51(\mathrm{~d}, J 16.5 \mathrm{~Hz}, 1 \mathrm{H}), 3.44-3.32(\mathrm{~m}, 3 \mathrm{H}), 3.14$ (dd, J 3.7, $10.4 \mathrm{~Hz}, 1 \mathrm{H}) .{ }^{13} \mathrm{C} \mathrm{NMR}(\mathrm{CDCl})_{3}, 125$ $\mathrm{MHz}) \delta 169.5,158.6,143.3,137.5,128.7,128.5,128.1,127.8,127.6,127.3,86.9,75.2,63.9,61.8,60.4,46.3$, 43.7. HRMS-ESI: $m / z[\mathrm{M}+\mathrm{Na}]^{+}$calcd for $\mathrm{C}_{33} \mathrm{H}_{32} \mathrm{~N}_{2} \mathrm{O}_{5} \mathrm{Na}^{+}: 559.2203$; Found: 559.2201

\section{$\mathbf{N}$-(4-Methoxybenzyl)-2-((4R*,5S*)-4-hydroxymethyl-2-oxo-5-trityloxymethyl-oxazolidin-3-yl)-acetamide}

(4f). Compound $\mathbf{4 f}$ (50 mg, 90\%) was obtained as a white solid from compound 8 (42 $\mathrm{mg}, 0.1 \mathrm{mmol}$ ) and 4methoxybenzyl amine $(27 \mathrm{mg}, 0.2 \mathrm{mmol})$ following General Procedure A; mp 121-124 ${ }^{\circ} \mathrm{C} ;{ }^{1} \mathrm{H} \mathrm{NMR}_{(\mathrm{CDCl}}, 500$ $\mathrm{MHz}) \delta 7.40-7.38(\mathrm{~m}, 6 \mathrm{H}), 7.30-7.26(\mathrm{~m}, 6 \mathrm{H}), 7.23-7.21(\mathrm{~m}, 3 \mathrm{H}), 7.16(\mathrm{~d}, J 8.5 \mathrm{~Hz}, 2 \mathrm{H}), 6.83(\mathrm{~d}, J 8.5 \mathrm{~Hz}, 2 \mathrm{H})$, $6.73(\mathrm{t}, J 5.5 \mathrm{~Hz}, 1 \mathrm{H}), 4.92(\mathrm{dd}, J 4.5,9.5 \mathrm{~Hz}, 1 \mathrm{H}), 4.60(\mathrm{q}, J 4.5 \mathrm{~Hz}, 1 \mathrm{H}), 4.37(\mathrm{~d}, J 5.5,14.5 \mathrm{~Hz}, 1 \mathrm{H}), 4.30-4.23(\mathrm{~m}$, $2 \mathrm{H}), 3.77(\mathrm{~s}, 3 \mathrm{H}), 3.71-3.68(\mathrm{~m}, 1 \mathrm{H}), 3.55-3.51(\mathrm{~m}, 2 \mathrm{H}), 3.44-3.38(\mathrm{~m}, 2 \mathrm{H}), 3.17(\mathrm{dd}, J 4.0,10.0 \mathrm{~Hz}, 1 \mathrm{H}) .{ }^{13} \mathrm{C} \mathrm{NMR}$ $\left(\mathrm{CDCl}_{3}, 125 \mathrm{MHz}\right) \delta 69.3,159.0,158.5,143.3,129.5,129.2,128.5,128.0,127.3,114.1,86.9,75.2,63.9,61.8$, $60.4,55.2,46.3,43.2$. HRMS-ESI: $\mathrm{m} / z[\mathrm{M}+\mathrm{Na}]^{+}$calcd for $\mathrm{C}_{35} \mathrm{H}_{36} \mathrm{~N}_{2} \mathrm{O}_{5} \mathrm{Na}^{+}: 587.2522$; Found: 587.2520

2-((4R*,5S*)-4-hydroxymethyl-2-oxo-5-trityloxymethyl-oxazolidin-3-yl)-N-(2-methoxyethyl)acetamide (4g). Compound $4 \mathrm{~g}$ (43 $\mathrm{mg} 87 \%$ ) was obtained as a white solid from compound 8 (42 $\mathrm{mg}, 0.1 \mathrm{mmol})$ and methoxyethylamine (15 mg, $0.2 \mathrm{mmol}$ ) following General Procedure A; mp 141-145 ${ }^{\circ} \mathrm{C} ;{ }^{1} \mathrm{H} \mathrm{NMR}_{(\mathrm{CDCl}}, 500$ $\mathrm{MHz})$ ठ 7.41-7.40 (m, 6H), 7.30-7.29 (m, 6H), 7.26-7.21 (m, 3H), $6.83(\mathrm{br} \mathrm{s}, 1 \mathrm{H}), 4.93(\mathrm{br} \mathrm{s}, 1 \mathrm{H}), 4.64(\mathrm{q}, J 4.5 \mathrm{~Hz}$, $1 \mathrm{H}), 4.35(\mathrm{~d}, J 17.0 \mathrm{~Hz}, 1 \mathrm{H}), 3.71-3.68(\mathrm{~m}, 1 \mathrm{H}), 3.54-3.46(\mathrm{~m}, 5 \mathrm{H}), 3.44-3.37(\mathrm{~m}, 3 \mathrm{H}), 3.35(\mathrm{~s}, 3 \mathrm{H}), 3.19(\mathrm{dd}, J 4.0$, $10.0 \mathrm{~Hz}, 1 \mathrm{H}) .{ }^{13} \mathrm{C} \mathrm{NMR}\left(\mathrm{CDCl}_{3}, 125 \mathrm{MHz}\right) \delta 169.5,158.5,143.3,128.6,128.0,127.3,86.9,74.9,70.7,63.8,62.0$, 60.3, 58.8, 46.8, 58.8, 46.8, 39.5. HRMS-ESI: $m / z[\mathrm{M}+\mathrm{Na}]^{+}$calcd for $\mathrm{C}_{29} \mathrm{H}_{32} \mathrm{~N}_{2} \mathrm{O}_{6} \mathrm{Na}^{+}:$527.2153; Found: 527.2156. 
N-(2-hydroxyethyl)-2-((4R*,5 $\left.S^{*}\right)$-4-hydroxymethyl-2-oxo-5-trityloxymethyl-oxazolidin-3-yl)acetamide (4h). Compound $4 \mathrm{~h}$ (43 $\mathrm{mg}, 89 \%$ ) was obtained as a white solid from compound 8 (42 $\mathrm{mg}, 0.1 \mathrm{mmol}$ ) and ethanolamine (12 mg, $0.2 \mathrm{mmol}$ ) following General Procedure A; mp 157-160 ${ }^{\circ} \mathrm{C} ;{ }^{1} \mathrm{H} \mathrm{NMR}\left(\mathrm{CDCl}_{3}, 500 \mathrm{MHz}\right) \delta$ 7.48-7.45 (m, 1H), 7.39-7.37 (m, 6H), 7.29-7.26 (m, 6H), 7.22-7.19 (m, 3H), 5.11 (br s, 1H), $4.61(\mathrm{q}, J 4.3 \mathrm{~Hz}, 1 \mathrm{H})$, $4.28(\mathrm{~d}, J 17.0 \mathrm{~Hz}, 1 \mathrm{H}), 4.92(\mathrm{br} \mathrm{s}, 1 \mathrm{H}), 3.69-3.57(\mathrm{~m}, 3 \mathrm{H}), 3.59(\mathrm{~d}, J 17.0 \mathrm{~Hz}, 1 \mathrm{H}), 3.48-3.47(\mathrm{~m}, 2 \mathrm{H}), 3.38-3.36$ $(\mathrm{m}, 2 \mathrm{H}), 3.18-3.17(\mathrm{~m}, 1 \mathrm{H}), 3.16(\mathrm{dd}, J 4.0,10.5 \mathrm{~Hz}, 1 \mathrm{H}) .{ }^{13} \mathrm{C} \mathrm{NMR}\left(\mathrm{CDCl}_{3}, 125 \mathrm{MHz}\right) \delta 170.0,158.9,143.3,128.5$, 128.0, 127.3, 86.9, 75.4, 63.9, 61.7, 61.0, 60.2, 46.4, 42.3. HRMS-ESI: $m / z[M+N a]^{+}$calcd for $\mathrm{C}_{28} \mathrm{H}_{30} \mathrm{~N}_{2} \mathrm{O}_{6} \mathrm{Na}^{+}$: 513.1996; Found: 513.2005

\section{2-((4R*,5 $\left.S^{*}\right)$-4-hydroxymethyl-2-oxo-5-trityloxymethyl-oxazolidin-3-yl)-N-(2-(methylamino)-2-}

oxoethyl)acetamide (4i). Compound $4 \mathbf{i}(86 \mathrm{mg}, 90 \%)$ was obtained as a white solid from compound 8 (80 mg, $0.19 \mathrm{mmol}$ ) and $\mathrm{N}$-methylglycinamide $(33 \mathrm{mg}, 0.38 \mathrm{mmol})$ following General Procedure A; mp $155-159{ }^{\circ} \mathrm{C} ;{ }^{1} \mathrm{H}$ $\mathrm{NMR}\left(\mathrm{CDCl}_{3}, 500 \mathrm{MHz}\right) \delta 7.76(\mathrm{t}, J 5.1 \mathrm{~Hz}, 1 \mathrm{H}), 7.40-7.39(\mathrm{~m}, 6 \mathrm{H}), 7.30-7.27(\mathrm{~m}, 6 \mathrm{H}), 7.24-7.21(\mathrm{~m}, 3 \mathrm{H}), 6.88(\mathrm{~d}$, J $4.1 \mathrm{~Hz}, 1 \mathrm{H}), 5.77(\mathrm{~s}, 1 \mathrm{H}), 4.62(\mathrm{q}, J 4.2 \mathrm{~Hz}, 1 \mathrm{H}), 4.34(\mathrm{~d}, J 17.0 \mathrm{~Hz}, 1 \mathrm{H}), 4.06(\mathrm{dd}, J 6.5,16.5 \mathrm{~Hz}, 1 \mathrm{H}), 3.78-3.72$ $(\mathrm{m}, 1 \mathrm{H}), 3.63(\mathrm{~d}, J 17.0 \mathrm{~Hz}, 1 \mathrm{H}), 3.56-3.55(\mathrm{~m}, 2 \mathrm{H}), 3.47-3.41(\mathrm{~m}, 2 \mathrm{H}), 3.18(\mathrm{dd}, J 3.3,10.3 \mathrm{~Hz}, 1 \mathrm{H}), 2.75(\mathrm{~d}, J 4.3$ $\mathrm{Hz}, 3 \mathrm{H})$. HRMS-ESI: $m / z$ [M+Na] ${ }^{+}$calcd for $\mathrm{C}_{29} \mathrm{H}_{31} \mathrm{~N}_{3} \mathrm{O}_{6} \mathrm{Na}^{+}: 540.2105$; Found: 540.2109.

Methyl (2-((4R*,5 $\left.S^{*}\right)$-4-hydroxymethyl-2-oxo-5-trityloxymethyl-oxazolidin-3-yl)acetyl)glycinate (4j). $50 \mathrm{mg}$ (96\%) of compound $\mathbf{4 j}$ was obtained as a white solid from compound 8 (42 $\mathrm{mg}, 0.1 \mathrm{mmol}$ ) and glycine methyl ester (18 mg, $0.2 \mathrm{mmol}$ ) following General Procedure A; mp $124-127{ }^{\circ} \mathrm{C} ;{ }^{1} \mathrm{H} \mathrm{NMR}\left(\mathrm{CDCl}_{3}, 500 \mathrm{MHz}\right) \delta 7.40-7.39$ $(\mathrm{m}, 6 \mathrm{H}), 7.30-7.28(\mathrm{~m}, 6 \mathrm{H}), 7.26-7.21(\mathrm{~m}, 4 \mathrm{H}), 4.62(\mathrm{q}, J 4.5 \mathrm{~Hz}, 1 \mathrm{H}), 4.41(\mathrm{~d}, J 17.0 \mathrm{~Hz}, 1 \mathrm{H}), 4.08(\mathrm{dd}, J 5.5,18.0$ $\mathrm{Hz}, 1 \mathrm{H}), 3.75-3.71(\mathrm{~m}, 1 \mathrm{H}), 3.71(\mathrm{~s}, 3 \mathrm{H}), 3.63(\mathrm{~d}, J 17.0 \mathrm{~Hz}, 1 \mathrm{H}), 3.58-3.55(\mathrm{~m}, 1 \mathrm{H}), 3.47-3.41(\mathrm{~m}, 2 \mathrm{H}), 3.17(\mathrm{dd}, J$ 4.0, $10.0 \mathrm{~Hz}, 1 \mathrm{H}) .{ }^{13} \mathrm{C} \mathrm{NMR}\left(\mathrm{CDCl}_{3}, 125 \mathrm{MHz}\right) \delta 170.7,169.9,158.7,143.3,128.6,128.1,127.4,57.0,75.1,63.8$, $61.9,60.4,52.6,46.8,41.1$. HRMS-ESI: $m / z[\mathrm{M}+\mathrm{Na}]^{+}$calcd for $\mathrm{C}_{29} \mathrm{H}_{30} \mathrm{~N}_{2} \mathrm{O}_{7} \mathrm{Na}^{+}:$541.1945; Found: 541.1947.

General Procedure B. Addition reaction of aziridine (3) and alkyl iodides. Fused-ring aziridine 5, Nal (200 mol\%) and the requisite alkyl iodide (200 mol\%) were dissolved in $\mathrm{CH}_{3} \mathrm{CN}(0.03 \mathrm{M})$ and the mixture was stirred while under reflux until the reaction was complete as determined by TLC. The solvent was removed and the product was purified via column chromatography (Ethyl acetate:hexanes; 1:2).

tert-Butyl 2-((4S*,5S*)-4-iodomethyl-2-oxo-5-trityloxymethyl-oxazolidin-3-yl)acetate (7a). Following General Procedure B, compound 7a (73 mg, 83\%) was obtained from compound 5 (52 mg, $0.14 \mathrm{~mol}$ ) and tert-butyl iodoacetate (68 mg, $0.28 \mathrm{mmol}$ ) as a white solid; $\mathrm{mp} 115-117{ }^{\circ} \mathrm{C} ;{ }^{1} \mathrm{H} \mathrm{NMR}\left(\mathrm{CDCl}_{3}, 500 \mathrm{MHz}\right) \delta=7.46-7.44(\mathrm{~m}$, $6 \mathrm{H}), 7.33-7.30(\mathrm{~m}, 6 \mathrm{H}), 7.26-7.23(\mathrm{~m}, 3 \mathrm{H}), 4.25(\mathrm{q}, J 5.0 \mathrm{~Hz}, 1 \mathrm{H}), 4.13(\mathrm{~d}, J 17.9 \mathrm{~Hz}, 1 \mathrm{H}), 4.11-4.10(\mathrm{~m}, 1 \mathrm{H}), 3.82$ (q, J $4.9 \mathrm{~Hz}, 1 \mathrm{H}), 3.70(\mathrm{~d}, J 8.0 \mathrm{~Hz}, 1 \mathrm{H}), 3.45(\mathrm{dd}, J 5.0,10.3 \mathrm{~Hz}, 1 \mathrm{H}), 3.37(\mathrm{dd}, J 4.8,10.4 \mathrm{~Hz}, 1 \mathrm{H}), 3.23(\mathrm{~d}, J 4.8$ $\mathrm{Hz}, 2 \mathrm{H}), 1.44$ (s, 9H). ${ }^{13} \mathrm{C} \mathrm{NMR}\left(\mathrm{CDCl}_{3}, 75 \mathrm{MHz}\right) \delta=167.2,157.2,143.3,128.6,128.0,127.3,87.2,82.7,78.7$, 63.9, 57.6, 44.1, 28.1, 6.9. HRMS-ESI: $\mathrm{m} / z$ [M+Na] ${ }^{+}$calcd for $\mathrm{C}_{30} \mathrm{H}_{32} \mathrm{INO}_{5} \mathrm{Na}^{+}: 636.1217$; Found: 636.1210 .

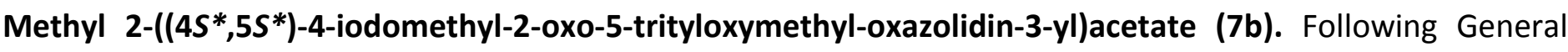
Procedure B, compound $7 \mathbf{b}(1.28 \mathrm{~g}, 90 \%)$ was obtained from compound 5 (0.93 g, 2.5 mol) and methyl iodoacetate (1.0 g, $5 \mathrm{mmol}$ ) as a white solid; $\mathrm{mp} 143-146{ }^{\circ} \mathrm{C} ;{ }^{1} \mathrm{H} \mathrm{NMR}\left(\mathrm{CDCl}_{3}, 300 \mathrm{MHz}\right) \delta=7.46-7.43(\mathrm{~m}, 6 \mathrm{H})$, 7.34-7.25 (m, 9H), 4.29-4.23 (m, 2H), 3.87-3.81 (m, 2H), 3.70 (s, 3H), $3.48(\mathrm{dd}, J 5.0,10.4 \mathrm{~Hz}, 1 \mathrm{H}), 3.34(\mathrm{dd}, J$ 4.5, $10.4 \mathrm{~Hz}, 1 \mathrm{H}), 3.23$ (d, J $4.9 \mathrm{~Hz}, 1 \mathrm{H}) .{ }^{13} \mathrm{C} \mathrm{NMR}\left(\mathrm{CDCl}_{3}, 75 \mathrm{MHz}\right) \delta=168.7,157.2,143.3,128.6,128.1,127.3$, 87.2, 78.9, 63.7, 57.5, 52.5, 43.4, 6.8. HRMS-ESI: $\mathrm{m} / z[\mathrm{M}+\mathrm{Na}]^{+}$calcd for $\mathrm{C}_{27} \mathrm{H}_{26} \mathrm{NO}_{5} \mathrm{Na}^{+}$: 594.0748; Found: 594.0744.

Ethyl 2-((4S*,5S*)-4-iodomethyl-2-oxo-5-trityloxymethyl-oxazolidin-3-yl)acetate (7c). Following General Procedure B, compound 7c (83 mg, 99\%) was obtained from compound 5 (52 mg, 0.14 mol) and ethyl iodoacetate (60 mg, $0.28 \mathrm{mmol}$ ) as a white solid; $\mathrm{mp} 135-138{ }^{\circ} \mathrm{C} ;{ }^{1} \mathrm{H} \mathrm{NMR}\left(\mathrm{CDCl}_{3}, 300 \mathrm{MHz}\right) \delta=7.46-7.43(\mathrm{~m}$, 
$6 \mathrm{H}), 7.34-7.24(\mathrm{~m}, 9 \mathrm{H}), 4.28-4.25(\mathrm{~m}, 4 \mathrm{H}), 3.85-3.82(\mathrm{~m}, 2 \mathrm{H}), 3.46(\mathrm{dd}, J 5.0,10.4 \mathrm{~Hz}, 1 \mathrm{H}), 3.36(\mathrm{dd}, J 4.5,10.4$ $\mathrm{Hz}, 1 \mathrm{H}), 3.23$ (d, J 4.8 Hz, 1H). ${ }^{13} \mathrm{C} \mathrm{NMR}\left(\mathrm{CDCl}_{3}, 75 \mathrm{MHz}\right) \delta=168.2,157.2,143.3,128.6,128.0,127.3,87.2,78.8$, $63.8,61.7,57.6,43.5,6.8$. HRMS-ESI: $m / z[\mathrm{M}+\mathrm{Na}]^{+}$calcd for $\mathrm{C}_{28} \mathrm{H}_{28} \mathrm{INO}_{5} \mathrm{Na}^{+}$: 608.0904; Found: 608.0899.

$\left(4 S^{*}, 5 S^{*}\right)$-4-lodomethyl-3-(2-oxo-2-phenylethyl)-5-trityloxymethyl-oxazolidin-2-one (7h). Following General

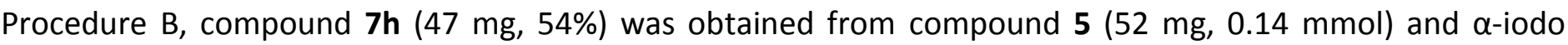
acetophenone (69 mg, $0.28 \mathrm{mmol}$ ) as a white solid; $\mathrm{mp} 107-109{ }^{\circ} \mathrm{C} ;{ }^{1} \mathrm{H} \mathrm{NMR}\left(\mathrm{CDCl}_{3}, 300 \mathrm{MHz}\right) \delta=7.96-7.93$ $(\mathrm{m}, 2 \mathrm{H})$, 7.64-7.59 (m, 1H), 7.51-7.46 (m, 8H), 7.34-7.22 (m, 10H), $4.96(\mathrm{~d}, J 18.2 \mathrm{~Hz}, 1 \mathrm{H}), 4.46(\mathrm{~d}, J 18.2 \mathrm{~Hz}, 1 \mathrm{H})$, $4.27(\mathrm{q}, J 5.0 \mathrm{~Hz}, 1 \mathrm{H}), 3.94-3.89(\mathrm{~m}, 1 \mathrm{H}), 3.52(\mathrm{dd}, J 5.1,10.4 \mathrm{~Hz}, 1 \mathrm{H}), 3.42(\mathrm{dd}, J 5.1,10.4 \mathrm{~Hz}, 1 \mathrm{H}), 3.25(\mathrm{dd}, J$ 6.0, $11.2 \mathrm{~Hz}, 1 \mathrm{H}), 3.18(\mathrm{dd}, J 3.2,11.2 \mathrm{~Hz}, 1 \mathrm{H}) .{ }^{13} \mathrm{C} \mathrm{NMR}\left(\mathrm{CDCl}_{3}, 75 \mathrm{MHz}\right) \delta=193.2,157.5,143.4,134.5,134.1$, 129.0, 128.7, 128.0, 127.3, 87.3, 79.0, 63.9, 57.6, 48.1, 7.3. HRMS-ESI: $m / z[\mathrm{M}+\mathrm{Na}]^{+}$calcd for $\mathrm{C}_{32} \mathrm{H}_{28} \mathrm{INO}_{4} \mathrm{Na}^{+}$: 640.0955; Found: 640.0950.

(4S*,5S*)-3-Benzyl-4-iodomethyl-5-trityloxymethyl-oxazolidin-2-one (7j). Following General Procedure B, compound 7j (57 mg, 87\%) was obtained from compound 5 (52 mg, $0.14 \mathrm{mmol}$ ) and benzyl iodide (61 mg, 0.28 $\mathrm{mmol})$ as a white solid; $\mathrm{mp} 112-115{ }^{\circ} \mathrm{C} ;{ }^{1} \mathrm{H} \mathrm{NMR}\left(\mathrm{CDCl}_{3}, 300 \mathrm{MHz}\right) \delta=7.38-7.35(\mathrm{~m}, 7 \mathrm{H}), 7.30-7.18(\mathrm{~m}, 13 \mathrm{H})$, 4.80 (d, J $15.4 \mathrm{~Hz}, 1 \mathrm{H}$ ), 4.22 (q, J $4.2 \mathrm{~Hz}, 1 \mathrm{H}), 4.10$ (d, J $15.2 \mathrm{~Hz}, 1 \mathrm{H}$ ), 3.49 (dd, J 4.3, $10.6 \mathrm{~Hz}, 1 \mathrm{H}), 3.33-3.28$ (m, $1 \mathrm{H}), 3.13-3.04(\mathrm{~m}, 2 \mathrm{H}), 2.98(\mathrm{dd}, J 2.6,11.0 \mathrm{~Hz}, 1 \mathrm{H})$; 157.6, 143.0, 137.1, 128.9, 128.6, 128.1, 128.0, 127.3, 90.1, 78.2, 63.3, 58.0, 47.3, 22.0.; HRMS-ESI: $\mathrm{m} / z[\mathrm{M}+\mathrm{Na}]^{+}$calcd for $\mathrm{C}_{31} \mathrm{H}_{28} \mathrm{INO}_{3} \mathrm{Na}^{+}$: 612.1006; Found: 612.1010.

General Procedure $\mathbf{C}$ for the addition reaction of aziridine $\mathbf{5}$ and alkyl bromides. The desired bromide (200 mol\%) was added to a solution of Compound 5 in $\mathrm{CH}_{3} \mathrm{CN}(0.03 \mathrm{M})$ and the reaction was stirred under reflux until the reaction was complete as determined by TLC. The solvent was removed and the product was purified via column chromatography (Ethyl acetate:hexanes; $1: 2$ ).

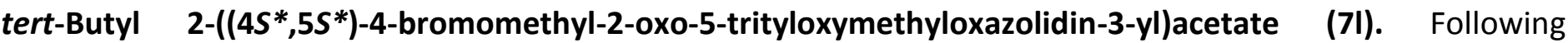
General Procedure C, compound $7 \mathbf{l}$ ( $52 \mathrm{mg}, 74 \%$ ) was obtained from compound 5 (52 mg, 0.14 mmol) and tertbutyl bromoacetate $(54.6 \mathrm{mg}, 0.28 \mathrm{mmol})$ as a white solid after column chromatography; mp $119-123{ }^{\circ} \mathrm{C} ;{ }^{1} \mathrm{H}$ NMR $\left(\mathrm{CDCl}_{3}, 300 \mathrm{MHz}\right) \delta=7.46-7.43(\mathrm{~m}, 6 \mathrm{H}), 7.34-7.22(\mathrm{~m}, 9 \mathrm{H}), 4.36(\mathrm{q}, 1 \mathrm{H}, J 5.0 \mathrm{~Hz}), 4.16-4.06(\mathrm{~m}, 2 \mathrm{H}), 3.78$ $(\mathrm{d}, J 18.0 \mathrm{~Hz}, 1 \mathrm{H}), 3.44-3.43(\mathrm{~m}, 4 \mathrm{H}) .{ }^{13} \mathrm{C} \mathrm{NMR}\left(\mathrm{CDCl}_{3}, 75 \mathrm{MHz}\right) \delta=167.3,157.2,143.3,128.6,128.0,127.3$, 87.2, 82.7, 76.6, 63.9, 58.1, 44.4, 32.1, 28.1. HRMS-ESI: $\mathrm{m} / z[\mathrm{M}+\mathrm{Na}]^{+}$calcd for $\mathrm{C}_{30} \mathrm{H}_{32} \mathrm{BrNO}_{5} \mathrm{Na}^{+}: 588.1356$; Found: 588.1352 .

(4S*,5S*)-3-Benzyl-4-bromomethyl-5-trityloxymethyloxazolidin-2-one (7n). Following General Procedure C, compound 7n (40 mg, 53\%) was obtained from compound 5 (52 mg, $0.14 \mathrm{mmol}$ ) and benzylbromide (47.9 mg, $0.28 \mathrm{mmol})$ as a white solid after column chromatography; mp 99-102 ${ }^{\circ} \mathrm{C} ;{ }^{1} \mathrm{H} \mathrm{NMR}\left(\mathrm{CDCl}_{3}, 300 \mathrm{MHz}\right) \delta=7.37-$ $7.36(\mathrm{~m}, 6 \mathrm{H}), 7.29-7.20(\mathrm{~m}, 14 \mathrm{H}), 4.80(\mathrm{~d}, J 15.4 \mathrm{~Hz}, 1 \mathrm{H}), 4.38(\mathrm{q}, J 3.9 \mathrm{~Hz}, 1 \mathrm{H}), 4.15(\mathrm{~d}, J 15.4 \mathrm{~Hz}, 1 \mathrm{H}), 3.61-3.60$ (m, $1 \mathrm{H}), 3.48(\mathrm{dd}, J 4.4,10.5 \mathrm{~Hz}, 1 \mathrm{H}), 3.28(\mathrm{dd}, J 6.0,11.2 \mathrm{~Hz}, 1 \mathrm{H}), 3.16-3.14(\mathrm{~m}, 1 \mathrm{H}), 3.11(\mathrm{dd}, J 3.3,10.5 \mathrm{~Hz}$,

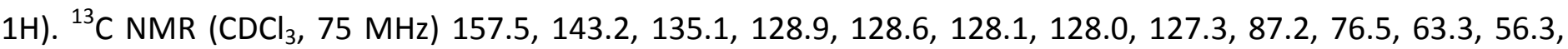
46.3, 32.3. HRMS-ESI: $\mathrm{m} / z$ [M+Na] ${ }^{+}$calcd for $\mathrm{C}_{31} \mathrm{H}_{28} \mathrm{BrNO}_{3} \mathrm{Na}^{+}: 564.1145$; Found: 564.1149.

$\left(1 S^{*}, 8 a R^{*}\right)$-1-(Trityloxymethyl)dihydro-1H,3H-oxazolo[4,3-c][1,4]oxazine-3,6(5H)-dione (8). Compound 9 $(2.40 \mathrm{~g}, 4.3 \mathrm{mmol})$ was dissolved in $100 \mathrm{~mL}$ of $\mathrm{CH}_{3} \mathrm{CN}$ and $\mathrm{Ag}_{2} \mathrm{O}(1.60 \mathrm{~g}, 6.9 \mathrm{mmol})$ was added. The mixture was stirred at RT for 2 hours and the solvent was removed. After column chromatography (Petroleum ether : Ethyl acetate, 2:1) compound 8 (1.62 g, 88\%) was obtained as a white solid; mp 136-139 ${ }^{\circ} \mathrm{C} ;{ }^{1} \mathrm{H} \mathrm{NMR}(\mathrm{CDCl}, 300$ $\mathrm{MHz}) \delta=4.56(\mathrm{~d}, 1 \mathrm{H}, J 19.2 \mathrm{~Hz}), 4.41(\mathrm{dd}, 1 \mathrm{H}, J 3.9,11.0 \mathrm{~Hz}), 4.29(\mathrm{t}, 1 \mathrm{H}, J 10.9 \mathrm{~Hz}), 4.17-4.08(\mathrm{~m}, 1 \mathrm{H}), 4.07(\mathrm{~d}$, $1 \mathrm{H}, \mathrm{J} 19.2 \mathrm{~Hz}$ ), 3.94-3.88 (m, 1H), 3.47 (dd, $1 \mathrm{H}, J$ 5.6, $10.4 \mathrm{~Hz}), 3.39$ (dd, $1 \mathrm{H}, \mathrm{J} 4.3,10.4 \mathrm{~Hz}) .{ }^{13} \mathrm{C} \mathrm{NMR}\left(\mathrm{CDCl}_{3}, 75\right.$ 
$\mathrm{MHz}) \delta=163.6,155.8,143.1,128.4,128.2,127.5,87.4,74.5,70.3,63.3,51.2,43.5 . \mathrm{HRMS}-\mathrm{ESI}: \mathrm{m} / \mathrm{z}[\mathrm{M}+\mathrm{Na}]^{+}$ calcd for $\mathrm{C}_{26} \mathrm{H}_{23} \mathrm{NO}_{5} \mathrm{Na}^{+}: 452.1468$; Found: 452.1470 .

2-((4S*,5S*)-4-lodomethyl-2-oxo-5-trityloxymethyl-oxazolidin-3-yl)acetic acid (9). Compound $7 \mathrm{~b}$ (448 mg, $0.78 \mathrm{mmol}$ ) was dissolved in $25 \mathrm{~mL}$ of THF and the solution was cooled to $0{ }^{\circ} \mathrm{C}$. $\mathrm{LiOH}(37 \mathrm{mg}, 1.56 \mathrm{mmol}$ ) was dissolved in $5 \mathrm{~mL}$ of water and the aqueous solution was added to the solution of ester $7 \mathbf{b}$. The reaction mixture was stirred at $0{ }^{\circ} \mathrm{C}$ for 2 hours and the reaction was concentrated. The remaining aqueous solution was acidified by $\mathrm{HCl}(0.1 \mathrm{M})$ to $\mathrm{pH} \mathrm{2}$, and the product was extracted with EtOAc $(3 \times 15 \mathrm{~mL})$. The organic layer was dried over $\mathrm{MgSO}_{4}$, filtered, concentrated and purified by column chromatography (Ethyl acetate:Petroleum ether, $1: 1)$. Compound 9 (370 mg, 85\%) was obtained as white oil; ${ }^{1} \mathrm{H} \mathrm{NMR}\left(\mathrm{CDCl}_{3}, 300\right.$ $\mathrm{MHz}) \delta=7.41-7.31(\mathrm{~m}, 6 \mathrm{H}), 7.29-7.19(\mathrm{~m}, 9 \mathrm{H}), 4.27-4.19(\mathrm{~m}, 2 \mathrm{H}), 3.86-3.74(\mathrm{~m}, 2 \mathrm{H}), 4.46(\mathrm{dd}, 1 \mathrm{H}, J 4.7,10.5$ $\mathrm{Hz}), 4.33(\mathrm{dd}, 1 \mathrm{H}, \mathrm{J} 4.4,10.5 \mathrm{~Hz}), 3.15(\mathrm{~d}, 1 \mathrm{H}, \mathrm{J} 4.7 \mathrm{~Hz}) .{ }^{13} \mathrm{C} \mathrm{NMR}\left(\mathrm{CDCl}_{3}, 75 \mathrm{MHz}\right) \delta=172.2,157.7,143.3,128.6$, 128.1, 127.4, 87.2, 79.2, 63.7, 57.6, 43.3, 6.7. HRMS-ESI: $\mathrm{m} / \mathrm{z}[\mathrm{M}+\mathrm{Na}]^{+}$calcd for $\mathrm{C}_{26} \mathrm{H}_{24} \mathrm{INO}_{5} \mathrm{Na}+: 580.059137$; Found: 580.059185 .

General procedure E. Reaction of compound 4 a with carboxylic acid using $\mathbf{N}, \boldsymbol{N}^{\prime}$-Dicyclohexylcarbodiimide (DCC). The requisite acid was dissolved in $\mathrm{CH}_{2} \mathrm{Cl}_{2}(0.03 \mathrm{M})$ then DCC (110 mol\%), DMAP (10 mol\%), and alcohol 4a (110 mol\%) was then added. The reaction mixture was stirred for 12 hours at $\mathrm{rt}$ and the precipitate was filtered off. The reaction was concentrated and purified via column chromatography $\left(\mathrm{CHCl}_{3}: \mathrm{PrOH}, 50: 1\right)$.

(3-(2-Methylamino-2-oxoethyl)-2-oxo-5-trityloxymethyl-oxazolidin-4-yl)methyl isobutyrate (10a). Following General Procedure E, compound 10a (61 mg, 81\%) was obtained from compound $4 \mathrm{a}$ (70 $\mathrm{mg}, 0.15 \mathrm{mmol})$ and isobutyric acid (15 mg, $0.17 \mathrm{mmol}$ ) as a white solid; $\mathrm{mp} 138-140{ }^{\circ} \mathrm{C} ;{ }^{1} \mathrm{H} \mathrm{NMR}\left(\mathrm{CDCl}_{3}, 500 \mathrm{MHz}\right) \delta=7.41-7.40$ $(\mathrm{m}, 6 \mathrm{H}), 7.34-7.32(\mathrm{~m}, 6 \mathrm{H}), 7.27-7.24(\mathrm{~m}, 3 \mathrm{H}), 6.28(\mathrm{br} \mathrm{s}, 1 \mathrm{H}), 4.36(\mathrm{q}, J 3.8 \mathrm{~Hz}, 1 \mathrm{H}), 4.27(\mathrm{dd}, J 3.5,12.5 \mathrm{~Hz}, 1 \mathrm{H})$, $4.10(\mathrm{~d}, J 17.0 \mathrm{~Hz}, 1 \mathrm{H}), 4.04(\mathrm{dd}, J 3.5,12.0 \mathrm{~Hz}, 1 \mathrm{H}), 3.86-3.83(\mathrm{~m}, 1 \mathrm{H}), 3.83(\mathrm{~d}, J 17.0 \mathrm{~Hz}, 1 \mathrm{H}), 3.62(\mathrm{dd}, J 4.0$, $10.5 \mathrm{~Hz}, 1 \mathrm{H}), 3.14$ (dd, J 3.0, $11.0 \mathrm{~Hz}, 1 \mathrm{H}), 2.53$ (sept, J 7.0, 1H), 2.45 (d, J 5.0, 3H), $1.12(\mathrm{~d}, J 7.0,6 \mathrm{H}) .{ }^{13} \mathrm{C} \mathrm{NMR}$ (Acetone, $500 \mathrm{MHz}$ ) $\delta=176.4,168.1,158.0,143.0,128.6,128.2,127.5,87.5,75.3,64.1,61.6,57.5,46.3,33.8$, $25.9,18.9,18.9$. HRMS-ESI: $m / z[\mathrm{M}+\mathrm{Na}]^{+}$calcd for $\mathrm{C}_{26} \mathrm{H}_{23} \mathrm{NO}_{5} \mathrm{Na}^{+}: 553.2309$; Found: 553.2312.

(3-(2-Benzylamino-2-oxoethyl)-2-oxo-5-trityloxymethyl-oxazolidin-4-yl)methyl benzoate (10b). Following General Procedure E, compound $10 \mathrm{~b}$ (53 mg, 85\%) was obtained from compound $4 \mathrm{a}$ ( $52 \mathrm{mg}, 0.10 \mathrm{mmol})$ and 4-pentynoic acid (11 mg, $0.11 \mathrm{mmol}$ ) as a white solid; $\mathrm{mp} 97-101{ }^{\circ} \mathrm{C} ;{ }^{1} \mathrm{H} \mathrm{NMR}\left(\mathrm{CDCl}_{3}, 500 \mathrm{MHz}\right) \delta=7.43$ 7.41(m, 6H), 7.34-7.31 (m, 6H), 7.28-7.25 (m, 3H), $6.25(\mathrm{q}, J 4.4,1 \mathrm{H}), 4.37(\mathrm{q}, J 3.9,1 \mathrm{H}), 4.10-4.04(\mathrm{~m}, 2 \mathrm{H})$, 3.87-3.84 (m, 2H), 3.60 (dd, J 3.9, $10.7 \mathrm{~Hz}, 1 \mathrm{H}), 3.16(\mathrm{dd}, J 3.2,10.7 \mathrm{~Hz}, 1 \mathrm{H}), 2.55-2.45(\mathrm{~m}, 7 \mathrm{H}), 1.96(\mathrm{t}, J 2.5 \mathrm{~Hz}$, $1 \mathrm{H}) .{ }^{13} \mathrm{C} \mathrm{NMR}\left(\mathrm{CDCl}_{3}, 125 \mathrm{MHz}\right) \delta=171.1,168.1,158.0,143.0,128.6,128.2,127.5,87.5,82.0,75.2,69.5,64.0$, 62.2, 57.5, 46.6, 33.1, 25.9, 14.3; HRMS-ESI: $m / z[\mathrm{M}+\mathrm{Na}]^{+}$calcd for $\mathrm{C}_{32} \mathrm{H}_{32} \mathrm{~N}_{2} \mathrm{O}_{6} \mathrm{Na}^{+}: 563.2153$; Found: 563.2154 .

(3-(2-(Methylamino)-2-oxoethyl)-2-oxo-5-trityloxymethyl-oxazolidin-4-yl)methyl $\quad \mathbf{N}$-(tert-butoxycarbonyl) glycinate (10c). Following General Procedure E, compound 10c (120 mg, 77\%) was obtained from compound 4a (110 mg, $0.20 \mathrm{mmol}$ ) and Boc-glycine (35 mg, $0.22 \mathrm{mmol})$ as a white solid; $\mathrm{mp} 198-202{ }^{\circ} \mathrm{C} ;{ }^{1} \mathrm{H} \mathrm{NMR}\left(\mathrm{CDCl}_{3}\right.$, $500 \mathrm{MHz}) \delta=7.41-7.40(\mathrm{~m}, 6 \mathrm{H}), 7.33-7.30(\mathrm{~m}, 6 \mathrm{H}), 7.27-7.26(\mathrm{~m}, 3 \mathrm{H}), 6.42(\mathrm{~s}, 1 \mathrm{H}), 5.28(\mathrm{~s}, 1 \mathrm{H}), 4.29-4.25(\mathrm{~m}$, 2H), 4.10 (dd, J 5.3, $12.0 \mathrm{~Hz}, 1 \mathrm{H}), 3.99-3.79(\mathrm{~m}, 5 \mathrm{H}), 3.56(\mathrm{~d}, J 10.5 \mathrm{~Hz}, 1 \mathrm{H}), 3.18(\mathrm{~d}, J 10.5 \mathrm{~Hz}, 1 \mathrm{H}), 2.55(\mathrm{~d}, J 4.5$ $\mathrm{Hz}, 3 \mathrm{H}), 1.44$ (s, 9H). ${ }^{13} \mathrm{C} \mathrm{NMR}\left(\mathrm{CDCl}_{3}, 125 \mathrm{MHz}\right) \delta=169.9,168.3,158.0,156.0,143.0,128.6,128.2,127.5,57.5$, 87.5, 80.2, 74.9, 63.9, 63.6, 57.5, 47.0, 42.4, 28.3, 26.0; HRMS-ESI: $m / z[\mathrm{M}+\mathrm{Na}]^{+}$calcd for $\mathrm{C}_{34} \mathrm{H}_{39} \mathrm{~N}_{3} \mathrm{O}_{8} \mathrm{Na}^{+}$: 640.2629; Found: 640.2627. 


\section{References}

1 Perez, J. J. Curr. Top. Med. Chem. 2018, 18, 566-590. https://doi.org/10.2174/1568026618666180522075258

2 Avan, I.; Hall, C. D.; Katritzky, A. R. Chem. Soc. Rev. 2014, 43, 3575-3594. https://doi.org/10.1039/c3cs60384a

3 Trabocchi, A.; Guarna, A. Peptidomimetics in Organic and Medicinal Chemistry; Wiley, 2014. https://doi.org/10.1002/9781118683033

4 Crowley, B. M.; Stump, C. A.; Nguyen, D. N.; Potteiger, C. M.; McWherter, M. A.; Paone, D. V.; Quigley, A. G.; Bruno, J. G.; Cui, D.; Culberson, J. C.; Danziger, A.; Fandozzi, C.; Gauvreau, D.; Kemmerer, A. L.; Menzel, K.; Moore, E. L.; Mosser, S. D.; Reddy, V.; White, R. B.; Salvatore, C. A.; Kane, S. A.; Bell, I. M.; Selnick, H. G.; Fraley, M. E.; Burgey, C. S. Bioorg. Med. Chem. Lett. 2015, 25, 4777-4781. https://doi.org/10.1016/j.bmcl.2015.07.021

5 De Marco, R.; Greco, A.; Rupiani, S.; Tolomelli, A.; Tomasini, C.; Pieraccini, S.; Gentilucci, L. Org. Biomol. Chem. 2013, 11, 4316-4326. https://doi.org/10.1039/c3ob40357b

6 Gentilucci, L.; Tolomelli, A.; De Marco, R.; Tomasini, C.; Feddersen, S. Eur. J. Org. Chem. 2011, 25, 49254930.

7 Ohta, Y.; Itoh, S.; Shigenaga, A.; Shintaku, S.; Fujii, N.; Otaka, A. Org. Lett. 2006, 8, 467-470. https://doi.org/10.1021/ol052755m

8 Tomasini, C.; Luppi, G.; Monari, M. J. Am. Chem. Soc. 2006, 128, 2410-2420. https://doi.org/10.1021/ja056762h

9 Elashal, H. E.; Raj, M. Chem. Commun. 2016, 52, 6304-6307. https://doi.org/10.1039/C6CC01509C

10 Orac, C. M.; Zhou, S.; Means, J. A.; Boehm, D.; Bergmeier, S. C.; Hines, J. V. J. Med. Chem. 2011, 54, 67866795.

\section{https://doi.org/10.1021/jm2006904}

11 Bergmeier, S. C.; Stanchina, D. M. J. Org. Chem. 1999, 64, 2852-2859. https://doi.org/10.1021/jo9823893

12 Anupam, R.; Nayek, A.; Green, N. J.; Grundy, F. J.; Henkin, T. M.; Means, J. A.; Bergmeier, S. C.; Hines, J. V. Bioorg. Med. Chem. Lett. 2008, 18, 3541-3544.

https://doi.org/10.1016/j.bmcl.2008.05.015

13 Means, J.; Katz, S. J.; Anupam, R.; Nayek, A.; Hines, J. V.; Bergmeier, S. C. Bioorg. Med. Chem. Lett. 2006, 16, 3600-3604.

https://doi.org/10.1016/j.bmcl.2006.03.068

14 Maciagiewicz, I.; Zhou, S.; Bergmeier, S. C.; Hines, J. V. Bioorg. Med. Chem. Lett. 2011, 21, 4524-4527. https://doi.org/10.1016/j.bmcl.2011.05.130

15 Hancock, M. T.; Pinhas, A. R. Tet. Lett. 2003, 44, 5457-5460. https://doi.org/10.1016/S0040-4039(03)01325-X

16 Nonn, M.; Remete, A. M.; Fulop, F.; Kiss, L. Tetrahedron 2017, 73, 5461-5483. https://doi.org/10.1016/i.tet.2017.07.062

17 Stankovic, S.; D'Hooghe, M.; Catak, S.; Eum, H.; Waroquier, M.; Van Speybroeck, V.; De Kimpe, N.; Ha, H.-J. Chem. Soc. Rev. 2012, 41, 643-665.

18 Lu, P. Tetrahedron 2010, 66, 2549-2560. 
https://doi.org/10.1016/i.tet.2010.01.077

19 Caille, J. C.; Farnier, M.; Guilard, R.; Aubry, A.; Lecomte, C. Can. J. Chem. 1986, 64, 831-836. https://doi.org/10.1139/v86-136

20 Jefford, C. W.; Sledeski, A. W.; Lelandais, P.; Boukouvalas, J. Tet. Lett. 1992, 33, 1855-1858. https://doi.org/10.1016/S0040-4039(00)74160-8

21 Still, W. C.; Kahn, M.; Mitra, A. J. Org. Chem. 1978, 43, 2923-2925. https://doi.org/10.1021/jo00408a041

22 Eliel, E. L.; Wilen, S. H. Stereochemistry of Organic Compounds; Wiley-Interscience, 1994. 